\section{Physical activity and erectile} dysfunction: a systematic review

\section{Atividade física e disfunção erétil: uma revisão sistemática}

\author{
Camila da Cruz Ramos de Araujo \\ Melissa de Carvalho Souza \\ Aline Renata Rentz Fernandes ${ }^{1}$ \\ Andreia Pelegrini' \\ Alexandro Andrade \\ Adriana Coutinho de Azevedo Guimarães ${ }^{1}$
}

\begin{abstract}
Defined as the constant or recurrent inability to attain or maintain a satisfactory erection, erectile dysfunction (ED) has multifactorial causes. Regular physical activity can be used as a preventive tool for ED. In this sense, the main objective of this study was to analyze the scientific literature, published in the last ten years (2004-2014), about the relationship between physical activity, ED and the associated factors among men older than 17 years in cross-sectional studies, randomized clinical trials and cohort studies. A systematic review was performed in articles published in the Bireme, ScienceDirect, and PubMed/Medline databases, in the SciELO electronic library and in the Portal of the Coordination for the Improvement of Higher Education Personnel (CAPES) Journal and written in Portuguese, English and Spanish. A total of 230 studies were identified, and only 19 met the inclusion criteria, all of which were published in English. ED was diagnosed in all studies. Concerning physical activity, 17 studies identified a significant association with a lower incidence of ED, including cross-sectional and quasi-experimental studies and randomized clinical trials. ED appeared to be associated with different factors related to men's lifestyle, particularly their level of education, non-communicable chronic diseases, body mass index and age.
\end{abstract}

\section{KEYWORDS}

Motor Activity; Men; Penile Erection; Sexual Health; Reproductive Health.

\begin{abstract}
RESUMO
Entendida como a incapacidade constante ou recorrente de alcançar ou manter uma ereção satisfatória, a disfunção erétil (DE) tem causas multifatoriais. A prática de atividade física pode ser utilizada como uma medida preventiva para a DE. Nesse sentido, o principal objetivo do presente estudo foi analisar a literatura cientifica, publicada nos últimos dez anos (2004 a 2014), sobre a relação entre atividade física, DE e os fatores associados em homens a partir de 17 anos e em estudos de corte transversal, ensaios clínicos randomizados e estudos de coorte. A análise dos artigos foi feita por meio de uma revisão sistemática de artigos publicados nas bases de dados Bireme, ScienceDirect, PubMed/ Medline, na biblioteca eletrônica SciELO e no Portal de Periódicos da Coordenadoria de Aperfeiçoamento de Pessoal de Nível Superior (CAPES) em português, inglês e espanbol. Identificou-se 230 estudos, sendo que apenas 19 preencheram os critérios de inclusão. Todos publicados em inglês. Em todos os estudos analisados, foi diagnosticada a presença de DE. No que diz respeito à atividade física, em 17 estudos, ela esteve significantemente associada à menor ocorrência de DE, tanto nos estudos com delineamento transversal quanto nos quase-experimentais e ensaios clinicos randomizados. $A D E$ pareceu estar associada a diferentes fatores relacionados ao estilo de vida do homem, nomeadamente, nível de escolaridade, doenças crônicas não transmissíveis, indice de massa corporal e também à idade.
\end{abstract}

\section{PALAVRAS-CHAVE}

Atividade motora; Homens; Ereção peniana; Saúde sexual; Saúde reprodutiva.
Rev Bras Ativ Fís Saúde p. 3-16 DOI

http://dx.doi.org/10.12820/rbafs.v.20n1p3

1 Universidade do Estado de Santa Catarina (UDESC) - Centro de Ciências da Saúde e do Esporte (CEFID), Florianópolis, SC, Brasil. 


\section{INTRODUCTION}

Erectile dysfunction (ED) can be defined as the constant or recurrent inability to attain or maintain a satisfactory erection in at least $50 \%$ of attempts of sexual intercourse ${ }^{1}$. Although not posing a risk to one's life, ED has a negative impact on self-esteem, quality of life and interpersonal relationships ${ }^{2}$. It is estimated that approximately 152 million men worldwide currently suffer from $\mathrm{ED}^{3}$ and that such prevalence is expected to reach nearly 322 million by $2025^{4}$. In a study conducted in Brazil, $45.1 \%$ of men had a certain level of ED, of which $31.2 \%$ were minimal; $12.2 \%$, moderate; and $1.7 \%$, complete 5 .

ED has multifactorial causes, which may be organic, psychogenic or mixed. Thus, it is possible to associate it with mental disorders ${ }^{6}$; hormonal disorders such as diabetes ${ }^{7}$, the reduction in testosterone levels ${ }^{8}$; hypertension ${ }^{9}$; smoking $^{10}$; alcoholism ${ }^{11}$, obesity ${ }^{12}$ and even cardiovascular diseases ${ }^{13}$, which are one of the most frequent causes ${ }^{14}$.

The literature indicates that ED is found in men aged 18 years and old$\mathrm{er}^{15-17}$ and it most frequently occurred between the ages of 40 and 70 years ${ }^{18}$. According to the Brazilian Urology Society, the prevalence of ED is $1 \%$ in men younger than 19 years; $3 \%$, in those aged 45 years; $6.7 \%$, in those aged between 45 and 55 years; and $25 \%$, in those aged until 75 years ${ }^{19}$. It could also be affirmed that age increases both the prevalence of ED and its severity ${ }^{5}$.

Several pharmacological and surgical advances have been made in the treatment of ED. In this sense, there has been an increase in the demand for specialized clinical services, as both health professionals and lay people are becoming more interested in this theme ${ }^{20}$. Studies indicate that, in addition to the specialized services and advances in the treatment of ED, physical activity has also been considered as a relevant treatment for this disease ${ }^{21-23}$, as it provides benefits to the cardiovascular system, such as the reduction in vascular diseases, improvement in endothelial functions and increase in blood flow in certain vascular beds ${ }^{24}$. These effects can stimulate penis vascularization ${ }^{25}$.

Apart from the above mentioned benefits, it is known that regular physical activity is also an important factor to prevent and fight the onset of certain diseases, such as diabetes ${ }^{26}$, heart diseases ${ }^{27}$, obesity ${ }^{28}$, depression ${ }^{29}$ and hypercholesterolemy $^{30}$, as these are associated with the development of ED ${ }^{22,31,32}$.

In this sense, physical activity and, consequently, a good physical condition can be considered as a preventive and therapeutic measure to control ED and diseases associated with a higher cardiovascular risk ${ }^{33}$. According to this perspective, the present study aimed to analyze the scientific literature published in the last ten years (from 2004 to 2014) regarding the relationship between physical activity, ED and associated factors in men aged 17 years and older found in cross-sectional studies, randomized clinical trials and cohort studies.

\section{METHODS}

The present study was characterized as a systematic review of research projects that analyzed the relationship between physical activity and ED. Aiming to display data in a more technical way, the recommendations from the Preferred Reporting Items for Systematic Reviews and Meta-analysis - PRIS$\mathrm{MA}^{34}$ were followed. Through an electronic search, articles from the follow- 
ing databases and electronic libraries and published in the last ten years (from 2004 to 2014) were selected: PubMed/Medline, Bireme, ScienceDirect, SciELO (Scientific Electronic Library Online) and (CAPES - Coordination for the Improvement of Higher Education Personnel). The search for studies was conducted between March and May 2014.

The key words used in this search and based on health science descriptors (DESCs) were as follows: "physical activity" and "erectile dysfunction", present in the titles, abstracts and key words. The AND Boolean operator was applied for term combination. The following search strategies were used in each database and electronic library: PubMed/Medline, "physical activity AND erectile dysfunction"; Bireme, "actividad fisica AND disfuncíon eréctil/ atividade física AND disfunção erétil"; ScienceDirect, "physical activity AND erectile dysfuncion"; SciELO, "atividade física AND disfunção erétil; and CAPES, "physical activity AND erectile dysfunction".

The following were considered as inclusion criteria: 1) articles published in Portuguese, English and Spanish; 2) articles including the previously mentioned descriptors; 3) articles available online; 4) articles with a cross-sectional design, randomized clinical trial, quasi-experimental design, cohort studies and prospective studies; 5) articles that studied individuals aged 17 years and older; and 6) articles that directly or indirectly used a particular measure to assess physical activity.

A secondary search was conducted in the bibliographical references of the selected articles, aiming to identify other sources not detected through the initial search. The following were excluded from this study: monographs, theses, dissertations, abstracts, systematic reviews and opinion-based articles. After excluding the articles that did not meet the inclusion criteria, apart from duplicate articles, all articles selected were filed to organize the reading.

To increase the reliability of this study, the entire process of search in the electronic databases, study selection, article reading and information gathering was performed by two researchers. This process of article search and selection was replicated by the same researchers independently and blindly and there were no divergences regarding the final selection of articles. These selected studies were subsequently catalogued according to: year, periodical, sample, methods, instruments and results.

The risk of bias in the studies included was assessed independently by the same two reviewers. The scale proposed by Downs and Black ${ }^{35}$ was used. This scale is comprised of 27 items that include communication, external validity, internal validity (bias), internal validity (confounding factors) and statistical power ${ }^{35}$, aimed at the methodological assessment of studies that performed an intervention. A score of zero (0) was assigned to each question if the article did not meet the requirements being assessed, while a score of one (1) was given if these requirements were met. The exception was question five, which is about the main confounding factors described in the articles, when a score of two (2) was assigned if the question was met by these articles. As a result, the maximum score that an article could achieve was 28 points. Among the studies that did not include an intervention, an adapted version of this scale was used, as previously adopted by international studies on systematic reviews ${ }^{36,37}$. According to this scale, questions 4, 8, 13 to $15,19,23$ and 24 were excluded because they referred to clinical trials. Thus, 19 items were assessed, totaling a maximum score of 20 points. 
The main data extracted from each study were as follows: the study design, the number of individuals, the instruments used to assess physical activity and $\mathrm{ED}$, the covariables and the main findings. Prevalences, means, standard deviations, odds ratio $(\mathrm{OR})$ and relative risk were adopted to show the study results.

With regard to ethical aspects, the present study was based on a bibliographical search that used public databases exclusively. Therefore, as no human beings were involved, this study did not have to be submitted and approved by a Research Ethics Committee.

\section{RESULTS}

The flow chart used to select the articles is shown in Figure 1. A total of 230 studies were identified, of which 29 were in the Bireme database, 43 in the portal of theJournal, 99 in PubMed, three in Scielo, 55 in ScienceDirect, and one in the references of articles. Thus, 43 duplicate studies were excluded and 187 titles and abstracts were assessed. After this process, 166 articles were excluded, of which 118 through the analysis of titles not related to reviews, 14 that were review studies, two that were opinion-based articles, 27 that were published out of the period of time defined in the methods, and five that only had the abstract available. Consequently, 21 studies remained and could be fully read subsequently. Out of these 21 studies, two were excluded because they were published in languages that had not been defined in the methods. Finally, 19 articles were included in this review.
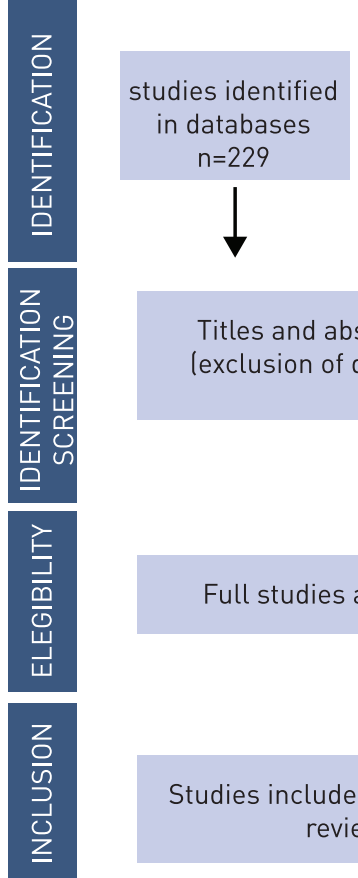

studies included

through references $\mathrm{n}=1$

Titles and abstracts assessed (exclusion of duplicates) $n=187$

two studies were excluded in the second analysis (published in languages not defined in the methods)

Full studies assessed $-n=21$

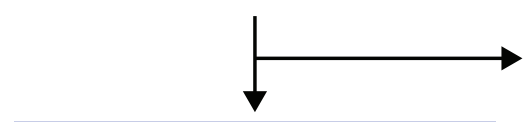

studies excluded according to title/abstract analyzed lat least one exclusion criterion) $n=166$

Studies included in the systematic review $n=19$

FIGURE 1 - Flow chart of the screening process and selection of articles to be included in the review.

The analysis of studies according to methodological procedures and results is shown in Chart 1 . Of all articles analyzed, the majority $(n=11)^{10,21,31-}$ $33,39,41,43,45-48$ were characterized as cross-sectional studies. 
CHART 1 - Type of research, sample, measures and results of studies on the relationship between physical activity and erectile dysfunction, published between 2004 and 2014 .

\begin{tabular}{|c|c|c|c|c|c|c|c|c|}
\hline Study (year) & Location & Design & Sample & PA Measure ${ }^{* * *}$ & ED Measure & Covariables & Results & Score* \\
\hline $\begin{array}{l}\text { Esposito, et } \\
\text { al. }(2004)^{25}\end{array}$ & Italy & $\begin{array}{l}\text { Randomized, } \\
\text { double-blind. }\end{array}$ & $\begin{array}{l}110 \text { men } \\
\text { aged } 35- \\
55 \text { years. }\end{array}$ & $\begin{array}{l}\text { Intervention: } \\
\text { Guidance to } \\
\text { increase the } \\
\text { level of physical } \\
\text { activity (walking; } \\
\text { swimming; } \\
\text { soccer) - } \\
\text { duration: } 2 \\
\text { years. }\end{array}$ & $\begin{array}{l}\text { International } \\
\text { Index of } \\
\text { Erectile } \\
\text { Function } \\
\text { (IIEF). }\end{array}$ & $\begin{array}{l}\text { Intervention: } \\
\text { Calorie intake } \\
\text { guidance } \\
\text { program - } \\
\text { duration: } 2 \text { years. }\end{array}$ & $\begin{array}{l}\text { After intervention, } \\
\text { there was a decrease in } \\
\text { body mass index (from } \\
36.9 \pm 2.5 \text { to } 31.2 \pm 2.1 \text { ), } \\
\text { increase in physical } \\
\text { activity level (from } \\
48 \pm 10 \text { to } 195 \pm 36 \text { min./ } \\
\text { week) and improvement } \\
\text { in the IIEF score (from } \\
13.9 \pm 4 \text { to } 17 \pm 5 \text { ) in the } \\
\text { intervention group. }\end{array}$ & $22^{* *}$ \\
\hline $\begin{array}{l}\text { Mirone, et } \\
\text { al. }(2004)^{31}\end{array}$ & Italy & Cross-sectional & $\begin{array}{l}12,558 \\
\text { men } \\
\text { aged } \\
\text { between } \\
17-98 \\
\text { years. }\end{array}$ & $\begin{array}{l}\text { Questions about } \\
\text { the frequency of } \\
\text { physical activity } \\
\text { per week. }\end{array}$ & $\begin{array}{l}\text { Structured } \\
\text { interview } \\
\text { about sexual } \\
\text { performance } \\
\text { to assess } \\
\text { erectile } \\
\text { function. }\end{array}$ & $\begin{array}{l}\text { Socio- } \\
\text { demographic } \\
\text { (personal } \\
\text { and lifestyle } \\
\text { characteristics } \\
\text { - age; weight; } \\
\text { height; marital } \\
\text { status; level of } \\
\text { education) and } \\
\text { clinical-historical } \\
\text { questionnaire. }\end{array}$ & $\begin{array}{l}\text { A total of } 19.9 \% \text { of men } \\
\text { were diagnosed with } \\
\text { ED. The frequency of ED } \\
\text { increased with age (from } \\
4.6 \% \text { in men aged less } \\
\text { than } 25 \text { years to } 37.6 \% \\
\text { in those aged more } \\
\text { than } 74 \text { years). Physical } \\
\text { activity for at least two } \\
\text { hours/week decreased } \\
\text { the risk of ED (OR 0.8, } \\
95 \% \mathrm{Cl}=0.7-0.9 \text { ) }\end{array}$ & $13 \#$ \\
\hline $\begin{array}{l}\text { Zheng, et al. } \\
(2006)^{32}\end{array}$ & China & Cross-sectional & 327 men. & $\begin{array}{l}\text { Structured } \\
\text { questions about } \\
\text { physical activity. }\end{array}$ & $\begin{array}{l}\text { Structured } \\
\text { questions } \\
\text { about erectile } \\
\text { function. }\end{array}$ & $\begin{array}{l}\text { Blood collection; } \\
\text { psychological } \\
\text { assessment; } \\
\text { socio- } \\
\text { demographic } \\
\text { questionnaire } \\
\text { lage; weight; } \\
\text { height; alcohol } \\
\text { and tobacco } \\
\text { consumption). }\end{array}$ & $\begin{array}{l}\text { A total of } 129 \text { men were } \\
\text { diagnosed with ED, } \\
\text { the risk of developing } \\
\text { ED was significantly } \\
\text { associated with low } \\
\text { levels of physical activity } \\
\text { (OR }=1.67,95 \% \mathrm{Cl}= \\
1.15-3.03) \text {. }\end{array}$ & $17 \#$ \\
\hline $\begin{array}{l}\text { Selvin, et al. } \\
(2007)^{39}\end{array}$ & USA & Cross-sectional & $\begin{array}{l}2,126 \\
\text { men } \\
\text { aged } \\
\text { more } \\
\text { than } 20 \\
\text { years. }\end{array}$ & $\begin{array}{l}\text { Questions about } \\
\text { physical activity. }\end{array}$ & $\begin{array}{l}\text { Individual } \\
\text { interview to } \\
\text { assess ED. }\end{array}$ & $\begin{array}{l}\text { Blood collection; } \\
\text { socio-demographic } \\
\text { information } \\
\text { lage; ethnic } \\
\text { group; marital } \\
\text { status; tobacco } \\
\text { consumption); } \\
\text { arterial pressure; } \\
\text { total cholesterol; } \\
\text { questions about } \\
\text { cardiovascular risk. }\end{array}$ & $\begin{array}{l}\text { There was a prevalence } \\
\text { of } 18.4 \% \text { of } \mathrm{ED} \text { in the } \\
\text { sample }(95 \% \mathrm{Cl}=16.2- \\
20.7) \text {. ED was } \\
\text { significantly associated } \\
\text { with low levels of } \\
\text { physical activity }(23.3 \% \\
95 \% \mathrm{Cl}=20.0-27.0) .\end{array}$ & $17 \#$ \\
\hline
\end{tabular}




\begin{tabular}{|c|c|c|c|c|c|c|c|c|}
\hline Study (year) & Location & Design & Sample & PA Measure ${ }^{* * *}$ & ED Measure & Covariables & Results & Score* \\
\hline $\begin{array}{l}\text { Revnic, et } \\
\text { al. }(2007)^{40}\end{array}$ & Romania & $\begin{array}{l}\text { Quasi- } \\
\text { experimental }\end{array}$ & $\begin{array}{l}42 \text { men } \\
\text { aged } \\
\text { between } \\
66-76 \\
\text { years. }\end{array}$ & $\begin{array}{l}16 \text {-week } \\
\text { physical activity } \\
\text { program. }\end{array}$ & $\begin{array}{l}\text { Clinical } \\
\text { assessment } \\
\text { to determine } \\
\text { ED. }\end{array}$ & $\begin{array}{l}\text { Geriatric } \\
\text { Depression } \\
\text { Scale (GDS); } \\
\text { electromyography } \\
\text { to assess muscle } \\
\text { strength. }\end{array}$ & $\begin{array}{l}\text { The physical activity } \\
\text { program improved } \\
\text { participants' } \\
\text { psychological and } \\
\text { physical aspects and } \\
\text { their erectile function. }\end{array}$ & $12^{* *}$ \\
\hline $\begin{array}{l}\text { Kratzik, et } \\
\text { al. }(2009)^{41}\end{array}$ & Austria & Cross-sectional & $\begin{array}{l}674 \text { men } \\
\text { aged } \\
\text { between } \\
45-60 \\
\text { years. }\end{array}$ & $\begin{array}{l}\text { Physical } \\
\text { activity through } \\
\text { Paffenbarger's } \\
\text { Score. }\end{array}$ & $\begin{array}{l}\text { International } \\
\text { Index of } \\
\text { Erectile } \\
\text { Function } \\
\text { (IIEF). }\end{array}$ & $\begin{array}{l}\text { Urological clinical } \\
\text { assessment; } \\
\text { endocrine } \\
\text { measures. }\end{array}$ & $\begin{array}{l}\text { Positive correlation } \\
\text { between the IIEF score } \\
\text { and Paffenbarger's } \\
\text { score ( } r=0.164, p< \\
0.001) . \text { The development } \\
\text { of ED decreased } 82.9 \% \\
\text { in active individuals } \\
(3,000 \mathrm{kcal} / \text { week), (OR = } \\
0.171, p=0.018) .\end{array}$ & $18 \#$ \\
\hline $\begin{array}{l}\text { Holden, et } \\
\text { al. }(2010)^{43}\end{array}$ & Australia & Cross-sectional & $\begin{array}{l}5,990 \\
\text { men } \\
\text { aged } \\
\text { more } \\
\text { than } 40 \\
\text { years. }\end{array}$ & $\begin{array}{l}\text { Questionnaire } \\
\text { about physical } \\
\text { activity (type, } \\
\text { intensity and } \\
\text { frequency in the } \\
\text { previous week). }\end{array}$ & $\begin{array}{l}\text { Questions } \\
\text { about sexual } \\
\text { function. }\end{array}$ & $\begin{array}{l}\text { Questions } \\
\text { about socio- } \\
\text { demographic } \\
\text { characteristics } \\
\text { lage; marital } \\
\text { status; } \\
\text { ethnic group; } \\
\text { occupation; level } \\
\text { of education; } \\
\text { weight; height; } \\
\text { alcohol and } \\
\text { tobacco } \\
\text { consumption) and } \\
\text { lifestyle. }\end{array}$ & $\begin{array}{l}\text { Individuals categorized } \\
\text { as inactive were more } \\
\text { likely to have ED (OR 1.5, } \\
95 \% \mathrm{Cl}=1.2-1.8 \text { ). }\end{array}$ & $16 \#$ \\
\hline $\begin{array}{l}\text { Kupelian, et } \\
\text { al. }(2010)^{44}\end{array}$ & USA & $\begin{array}{l}\text { Epidemiological } \\
\text { study }\end{array}$ & $\begin{array}{l}2,301 \\
\text { men } \\
\text { aged } \\
\text { between } \\
30-79 \\
\text { years. }\end{array}$ & $\begin{array}{l}\text { Physical Activity } \\
\text { Scale for the } \\
\text { Elderly (PASE). }\end{array}$ & $\begin{array}{l}\text { International } \\
\text { Index of } \\
\text { Erectile } \\
\text { Function } \\
\text { (IIEF). }\end{array}$ & $\begin{array}{l}\text { Questionnaire } \\
\text { about socio- } \\
\text { demographic } \\
\text { characteristics } \\
\text { lethnic group; } \\
\text { socioeconomic } \\
\text { level; level of } \\
\text { education; BMI). }\end{array}$ & $\begin{array}{l}\text { Lifestyle was associated } \\
\text { with age and ED was } \\
\text { associated with all study } \\
\text { covariables and physical } \\
\text { activity. }\end{array}$ & $12 \#$ \\
\hline $\begin{array}{l}\text { La Vignera, } \\
\text { et al. } \\
(2011)^{23}\end{array}$ & Italy & $\begin{array}{l}\text { Quasi- } \\
\text { experimental }\end{array}$ & $\begin{array}{l}50 \text { men } \\
\text { aged } \\
\text { between } \\
48-62 \\
\text { years. }\end{array}$ & $\begin{array}{l}\text { Protocol of } \\
\text { aerobic physical } \\
\text { activity (150min/ } \\
\text { week). }\end{array}$ & $\begin{array}{l}\text { International } \\
\text { Index of } \\
\text { Erectile } \\
\text { Function } \\
\text { (IIEF). }\end{array}$ & $\begin{array}{l}\text { Blood collection } \\
\text { (total cholesterol; } \\
\text { triglycerides; HDL } \\
\text { cholesterol). }\end{array}$ & $\begin{array}{l}\text { After three months of } \\
\text { aerobic physical activity, } \\
\text { the intervention group } \\
\text { had a significantly } \\
\text { higher IIEF score, } \\
\text { showing a lower } \\
\text { prevalence of ED (from } \\
11.0 \pm 1.0 \text { to } 16.5 \pm 1.0 \text { ). }\end{array}$ & $16^{* *}$ \\
\hline $\begin{array}{l}\text { Agostini, et } \\
\text { al. }(2011)^{33}\end{array}$ & Brazil & Cross-sectional & $\begin{array}{l}180 \text { men } \\
\text { aged } \\
\text { between } \\
40-75 \\
\text { years. }\end{array}$ & $\begin{array}{l}\text { International } \\
\text { Physical Activity } \\
\text { Questionnaire } \\
\text { (IPAQ-short } \\
\text { version). }\end{array}$ & $\begin{array}{l}\text { International } \\
\text { Index of } \\
\text { Erectile } \\
\text { Function } \\
\text { (IIEF-short } \\
\text { version). }\end{array}$ & $\begin{array}{l}\text { Anthropometric } \\
\text { measures; } \\
\text { physical fitness } \\
\text { measure (VO2 } 2_{\text {max }} \text { ); } \\
\text { assessment of } \\
\text { cardiac frequency } \\
\text { at rest; socio- } \\
\text { demographic } \\
\text { questionnaire. }\end{array}$ & $\begin{array}{l}\text { The majority of } \\
\text { individuals who had ED } \\
\text { were inactive }(95.6 \%) \\
\text { whereas the minority } \\
\text { were highly active }(9.1 \%) \\
\text { - p<0.001. }\end{array}$ & $15 \#$ \\
\hline
\end{tabular}




\begin{tabular}{|c|c|c|c|c|c|c|c|c|}
\hline Study (year) & Location & Design & Sample & PA Measure ${ }^{* * *}$ & ED Measure & Covariables & Results & Score* \\
\hline $\begin{array}{l}\text { Pohjantähti- } \\
\text { Maaroos, et } \\
\text { al. }(2011)^{45}\end{array}$ & Finland & Cross-sectional & $\begin{array}{l}120 \text { men } \\
\text { with a } \\
\text { mean } \\
\text { age of } 50 \\
\text { years. }\end{array}$ & $\begin{array}{l}\text { Structured } \\
\text { questionnaire } \\
\text { about physical } \\
\text { activity (type, } \\
\text { intensity and } \\
\text { frequency). }\end{array}$ & $\begin{array}{l}\text { International } \\
\text { Index of } \\
\text { Erectile } \\
\text { Function } \\
\text { (IIEF). }\end{array}$ & $\begin{array}{l}\text { Laboratory } \\
\text { exams. }\end{array}$ & $\begin{array}{l}\text { Individuals who } \\
\text { practiced physical } \\
\text { activity regularly (>kcal/ } \\
\text { day) were less likely } \\
\text { to have ED (OR 0.12, } \\
95 \% \mathrm{Cl}=0.017-0.778, \mathrm{p}= \\
0.027 \text { ). }\end{array}$ & $17 \#$ \\
\hline $\begin{array}{l}\text { Liao, et al } \\
(2012)^{46}\end{array}$ & China & Cross-sectional & $\begin{array}{l}1,776 \\
\text { men } \\
\text { aged } \\
\text { between } \\
20-77 \\
\text { years. }\end{array}$ & $\begin{array}{l}\text { Questions about } \\
\text { the weekly } \\
\text { practice of } \\
\text { physical activity. }\end{array}$ & $\begin{array}{l}\text { International } \\
\text { Index of } \\
\text { Erectile } \\
\text { Function } \\
\text { (IIEF). }\end{array}$ & $\begin{array}{l}\text { Anthropometric } \\
\text { measures; } \\
\text { blood collection } \\
\text { (triglycerides; } \\
\text { HDL cholesterol); } \\
\text { tobacco } \\
\text { consumption. }\end{array}$ & $\begin{array}{l}\text { The prevalence of ED } \\
\text { was } 47.6 \% \text {. ED was } \\
\text { more prevalent in } \\
\text { individuals who were } \\
\text { older, smokers and } \\
\text { hypertensive. }\end{array}$ & $19 \#$ \\
\hline $\begin{array}{l}\text { Wu, et al. } \\
(2012)^{10}\end{array}$ & China & Cross-sectional & $\begin{array}{l}2,686 \\
\text { men } \\
\text { aged } \\
\text { between } \\
17-88 \\
\text { years. }\end{array}$ & $\begin{array}{l}\text { Questionnaire to } \\
\text { assess physical } \\
\text { activity level. }\end{array}$ & $\begin{array}{l}\text { International } \\
\text { Index of } \\
\text { Erectile } \\
\text { Function } \\
\text { (IIEF). }\end{array}$ & $\begin{array}{l}\text { Questionnaire } \\
\text { about smoking } \\
\text { habit; BMI; blood } \\
\text { collection (total } \\
\text { cholesterol; HDL } \\
\text { cholesterol; } \\
\text { triglycerides). }\end{array}$ & $\begin{array}{l}\text { The prevalence of ED } \\
\text { was } 49.5 \% \text {. Individuals } \\
\text { who did not practice } \\
\text { physical activity } \\
\text { had a higher risk of } \\
\text { developing ED (OR 1.13, } \\
95 \% \mathrm{Cl}=0.97-1.32 \text { ) }\end{array}$ & $15 \#$ \\
\hline $\begin{array}{l}\text { Garimella, } \\
\text { et al. } \\
(2013)^{47}\end{array}$ & USA & Cross-sectional & $\begin{array}{l}5,994 \\
\text { men } \\
\text { aged } \\
\text { more } \\
\text { than } 65 \\
\text { years. }\end{array}$ & $\begin{array}{l}\text { Physical Activity } \\
\text { Scale for the } \\
\text { Elderly (PASE). }\end{array}$ & $\begin{array}{l}\text { International } \\
\text { Index of } \\
\text { Erectile } \\
\text { Function } \\
\text { (IIEF). }\end{array}$ & $\begin{array}{l}\text { Anthropometric } \\
\text { measures; socio- } \\
\text { demographic } \\
\text { questionnaire } \\
\text { lage; level of } \\
\text { education; } \\
\text { marital status; } \\
\text { ethnic group). }\end{array}$ & $\begin{array}{l}\text { Prevalence of moderate } \\
\text { to severe ED was } 56 \% \text {. } \\
\text { There was a relationship } \\
\text { between ED and body } \\
\text { mass index. }\end{array}$ & 19\# \\
\hline $\begin{array}{l}\text { Banks, et al. } \\
(2013)^{13}\end{array}$ & Australia & Cohort study & $\begin{array}{l}95,038 \\
\text { men } \\
\text { aged } \\
\text { more } \\
\text { than } 45 \\
\text { years. }\end{array}$ & $\begin{array}{l}\text { Questions about } \\
\text { physical activity. }\end{array}$ & $\begin{array}{l}\text { Questions } \\
\text { about sexual } \\
\text { health. }\end{array}$ & $\begin{array}{l}\text { Questions } \\
\text { about socio- } \\
\text { demographic } \\
\text { information, } \\
\text { lifestyle, body } \\
\text { mass index, } \\
\text { clinical history, } \\
\text { functional } \\
\text { capacity. }\end{array}$ & $\begin{array}{l}\text { Men with high levels } \\
\text { of physical activity had } \\
\text { lower ED indices. }\end{array}$ & $16 \#$ \\
\hline $\begin{array}{l}\text { Weber, et al. } \\
(2013)^{48}\end{array}$ & Australia & Cross-sectional & $\begin{array}{l}108,477 \\
\text { men } \\
\text { aged } \\
\text { more } \\
\text { than } 45 \\
\text { years. }\end{array}$ & $\begin{array}{l}\text { Medical } \\
\text { Outcomes } \\
\text { Study Physical } \\
\text { Functioning } \\
\text { Scale (MOS-PF). }\end{array}$ & $\begin{array}{l}\text { Interview to } \\
\text { assess EF. }\end{array}$ & $\begin{array}{l}\text { Questionnaire } \\
\text { about socio- } \\
\text { demographic } \\
\text { characteristics } \\
\text { (place of residence; } \\
\text { marital status; } \\
\text { level of education, } \\
\text { presence of } \\
\text { disease). }\end{array}$ & $\begin{array}{l}\text { The higher the physical } \\
\text { activity level, the lower } \\
\text { the occurrence of ED } \\
\text { (OR } 0.47 ; 95 \% \mathrm{Cl}=0.43 \text { - } \\
0.50 \text { ). }\end{array}$ & $16 \#$ \\
\hline
\end{tabular}

* Downs and Black's methodological quality scale score35

** Downs and Black's total scale score

\#Downs and Black's adapted scale score

*** PA: physical activity

With regard to the locations where the studies were performed, most of them originated from the United States ${ }^{38,39,42,44,47}$, followed by China ${ }^{10,21,32,46}$ and only one from Brazil ${ }^{33}$.

In terms of the instruments used to measure $\mathrm{ED}$, the one most frequently adopted was the International Index of Erectile Function (IIEF) $(\mathrm{n}=11)^{10,13,21,23,25,33,41,44-47}$. Different instruments were used to analyze physical activity, such as the IPAQ-short version ${ }^{33}$; closed questions about physical activity $^{10,13,21,31,39,42,46,47}$, the Physical Activity Scale for the Elderly (PASE) ${ }^{44,47}$; the Questionnaire about Lifestyle Factors ${ }^{43}$; Paffenberger's Physical Activi- 
ty Questionnaire ${ }^{41}$; and also three studies that performed interventions with physical activity programs ${ }^{23,25,40}$.

Among all 19 studies assessed, the presence of ED was found to be directly associated with lifestyle characteristics of the populations studied, such as alcohol ${ }^{31,38,42,46,48}$ and tobacco consumption ${ }^{10,13,21,33,38,39,43}$; the presence of certain diseases, mainly arterial hypertension ${ }^{10,25,31,32,39,41-43,46,47}$, diabetes ${ }^{10,13,31-}$ $33,39,42,43,47,48$ and cardiovascular diseases ${ }^{13,31,33,39,42,43,47,48}$. With regard to the age group of occurrence of ED, this condition was recurrent in individuals aged

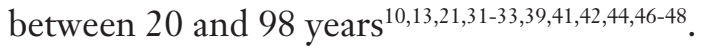

A total of 17 studies showed a significant association between physical activity and lower levels of $\mathrm{ED}^{10,13,21,23,25,31,32,33,38-45,48}$ and only two studies did not find significant associations between these variables ${ }^{46,47}$.

In quasi-experimental studies and randomized clinical trials, there was a significant improvement in ED levels after a period of physical activity intervention. This occurred when the same group was compared in the beginning and in the end of this program, as well as when the intervention and control groups were compared. With regard to cross-sectional studies, the associations between ED and physical activity were observed when considering the physical activity level primarily. The majority of studies reported that the higher the physical activity level, the lower the occurrence of ED. Additionally, physical activity also had a positive effect on ED in cohort and prospective studies.

Regarding the methodological assessment of review articles, the score found in studies on interventions varied between 12 and 22 points, with a mean of 17 points. In addition, the main gaps were observed in components associated with communication, external and internal (bias) validity, as well as not showing the actual probability values, blinding attempts and random selection of participants. Moreover, with regard to the studies that did not perform intervention, the score varied from 12 to 19 points, with a mean of 15.5 points.

Additionally, it was observed that gaps were also associated with communication aspects and how actual probability and internal validity (bias) values were shown, referring to the results based on analyses not defined a priori.

\section{DISCUSSION}

\section{Erectile dysfunction and associated factors}

Based on the literature analyzed in this review, a high prevalence of ED was found. In one of the studies, $56 \%$ of men had a moderate to severe level of $\mathrm{ED}^{47}$. With regard to a possible relationship between ED and physical activity, 17 studies revealed that physical activity was associated with a lower prevalence of such dysfunction.

In order to assess the presence and severity of ED, the majority of studies analyzed used the IIEF $(\mathrm{n}=11)^{10,13,21,23,25,33,41,44-47}$. Other studies applied an interview $^{31,39,48}$, clinical assessment ${ }^{40}$, questionnaires ${ }^{32,38,42}$ and telephone interviews $^{43}$ to conduct this evaluation. In the majority of these studies, the severity of ED symptoms was categorized as severe, moderate, minimal and none $\mathrm{e}^{10,13,23,33,41,44,46-48}$.

When the erectile function was assessed through a single question, namely "How would you describe your ability to attain and maintain an adequate 
erection for satisfactory sexual intercourse?”, the categorization of symptoms varied from "always capable" or "almost always capable", "usually capable", "sometimes capable" to "never capable" 39 ; very poor, poor, fair, good and very $\operatorname{good}^{38}$; and sometimes (moderate) or never (severe) ${ }^{43}$ capable of obtaining and maintaining an erection considered satisfactory for sexual intercourse. Furthermore, some studies categorized individuals into with or without $\mathrm{ED}^{21,25,31,32,42,45}$ exclusively, and only one study ${ }^{40}$ identified the presence or absence of ED through a clinical assessment.

The occurrence of ED affected individuals aged between 20 and 88 years, ranging from $18.4 \%$ to $49.5 \%$ of this population. Older men showed a higher prevalence of $\mathrm{ED}$, indicating a positive association with age in the majority of studies $^{10,13,21,31-33,38,39,41,43-45}$. According to Weber, Smith, O'Connell . (2013) ${ }^{48}$, as age advances, there is an annual increase in $11.3 \%$ in the chance of developing $\mathrm{ED}$, and the occurrence of ED is one in every six men between the ages of 40 and 70 years $^{49}$. These findings can be the result of the natural aging process, which includes, among other things, a steady reduction in testosterone levels in the blood that in its turn influences the development of ED and other sexual dysfunctions ${ }^{46}$.

In the studies analyzed, ED was also positively associated with the occurrence of certain diseases, among which the following were the most frequently mentioned: arterial hypertension ${ }^{10,25,31,32,39,41-43,46,47}$, diabetes ${ }^{10,13,31,32,39,42,43,47,48}$ and cardiovascular diseases ${ }^{13,31,39,42,43,47,48}$. According to Galvão and Chagas $(2005)^{50}$, the risk factors for cardiovascular diseases and ED can be considered as similar, so that the greater the occurrence of cardiovascular conditions, the higher the prevalence of ED. Such dysfunction is the initial manifestation in patients with cardiovascular diseases ${ }^{50}$ and diabetes ${ }^{51}$. Moreover, with regard to diseases, hyperglycemia, dyslipidemia, prostate cancer and hypercholesterolemia were found to be associated with the occurrence of $\mathrm{ED}^{31,33,46,47}$, apart from psychological disorders such as anxiety and depression ${ }^{32,43,48}$. According to Moreira, Abdo and Torres . $(2001)^{17}$, these disorders are associated with the occurrence of ED as they can lead to the reduction in sexual libido.

Unhealthy life habits such as smoking and alcohol drinking were also associated with $\mathrm{ED}^{31,38,42,46,48}$. However, when analyzed independently, some studies reported a positive association with tobacco consumption ${ }^{10,13,21,33,38,39,43}$ exclusively. With regard to alcohol consumption, Banks, Joshy, Abhayaratna . $(2013)^{13}$ found a positive association with ED and suggested that this occurs due to the fact that alcohol reduces plasma testosterone and increases serum estrogen, which may harm the erectile function in the long term ${ }^{52}$.

According to Bacon et al. (2006) $)^{38}$, these data are important to motivate individuals to adopt a healthier lifestyle, including physical activity and a balanced diet, thus avoiding an immediate problem. It should be emphasized that many men do not adopt such behavior as they argue that negative aspects for one's health and wellbeing only occur in older individuals ${ }^{38}$.

The Body Mass Index (BMI) was also observed as a determining factor for the development of ED. A high BMI was associated with a greater occurrence of $\mathrm{ED}^{25,32,38,39,41,42,47,48}$, as obesity directly affects the circulatory system in general, including the blood flow in the penile vessels ${ }^{47}$. Additionally, in the studies conducted by Cheng and $\mathrm{Ng}(2007)^{21}$ and Holden, McLachlan, Pitts . (2010) ${ }^{43}$, apart from obesity, low weight can also lead to a higher risk of development of ED as it is connected to the reduction in blood testosterone. 
The prevalence of ED was also associated with level of education ${ }^{10,13,31,39,42,48}$. According to Abdo, Oliveira Jr, Scanavino (2006) $)^{5}$, individuals with a low level of education have lower access to health care and less knowledge about the sexual function and what is harmful to sexual performance. It should be emphasized that these factors vary according to the environment and culture in which individuals are included.

In this sense, ED prevention is required through interventions that promote changes in lifestyle, such as smoking cessation, reduction in alcohol consumption and body weight control, in addition to encouraging the adequate treatment of cardiovascular diseases, diabetes and hypertension. Moreover, regular physical activity seems to play a very important role.

\section{Physical activity and erectile dysfunction}

Physical activity appeared to be directly associated with ED in 17 studies and the majority of them showed that such practice was also associated with a lower risk of development of ED. In one of the studies, categorized as cross-sectional, individuals considered to be active were found to have significantly lower levels of ED than those who were inactive or insufficiently active ${ }^{33}$. Following the same methodological design, similar results were obtained by Pohjantähti-Maaroos, Palomäki, Hartikainen $(2011)^{45}$, revealing that ED was less frequent in active individuals, thus enabling researchers to consider regular physical activity as a protective factor against the development of ED ${ }^{45}$.

Despite the differences in methodological designs, namely cohort $^{38}$, cross-sectional $^{19,41}$ and quasi-experimental studies ${ }^{40}$, the intensity of physical activity was also found to influence ED levels, with the greatest benefits resulting from moderate ${ }^{40}$ to vigorous ${ }^{38,39,41}$ levels of activity. According to Kratzik, Lackner, Mark . (2009) ${ }^{41}$, the intensity, type, duration and frequency of physical activity are factors that must be considered for the treatment of ED. In the cross-sectional study conducted by Mirone $(2004)^{31}$, men who practiced at least 120 minutes of weekly physical activity had a lower risk of developing ED.

With regard to the type of activity, quasi-experimental studies showed that aerobic physical activity was effective in the reduction of ED levels in individuals who previously had high levels ${ }^{23}$. Similar results were observed with walking, climbing stairs as a means of transport, and leisure sports ${ }^{41}$. These activities are associated with an improvement in erectile function, as they can provide physical and mental benefits, such as better endothelial function and vascularization and lower depressive state $^{43}$.

Studies have shown that physical activity was associated with ED according to the BMI of certain populations ${ }^{21,23,38,43,44}$. Physical activity was found to influence the risk of developing ED in individuals with a higher BMI. In some cases, obese men with low levels of physical activity had a higher risk of development of ED when compared to those with an average BMI and same levels of physical activity ${ }^{21,38}$. In their study, Bacon, Mittleman, Kawachi . (2006) ${ }^{38}$ emphasize that obesity and excessive body weight increase the risk of ED at any level of physical activity.

It should be noted that this association was not exclusive among men with a high BMI. Among the studies analyzed, eutrophic and underweight men who did not perform any physical activity also had a higher risk of develop- 
ment of ED ${ }^{21,43}$. According to Holden, McLachlan, Pitts . (2010) ${ }^{43}$, this finding can be justified by the fact that low weight can harm a man's body, including a significant reduction in blood testosterone levels, which can cause other male sexual function disorders, apart from the higher risk of ED. In this sense, it could be affirmed that the body weight of insufficiently active men can be a risk factor for the development of $\mathrm{ED}^{21}$.

Only two studies ${ }^{46,47}$ did not find significant associations between physical activity and ED levels. This response must be due to the fact that the majority of the sample studied was aged between 30 and 39 years, when ED is less frequent and severe ${ }^{10}$. However, interventions associated with physical activity should be included in this stage of life, considering the evidence that indicates a steady increase in ED with age $\mathrm{e}^{31,32,39}$.

The present review study had some limitations. The first one was associated with the way articles were obtained. Data could have been collected using other inclusion criteria. It should be emphasized that the methodology adopted by the articles to assess physical activity had some limitations, as the majority evaluated physical activity through questionnaires. In such cases, participants can overestimate this behavior as it is a practice considered to be desirable ${ }^{54}$. Additionally, it should be noted that this review only identified three cohort studies, two quasi-experimental studies and one clinical trial, whereas the majority of studies had a cross-sectional observational design. This gap could be due to the fact that the latter more easily include a great number of individuals. However, the results may not be as reliable as those found in cohort studies and randomized clinical trials.

Finally, a lack or low level of physical activity can be considered to be predictors of $\mathrm{ED}^{21}$. In this sense, changes in lifestyle through the promotion of physical exercising can lead to a reduction in $\mathrm{ED}^{25}$. Taking these arguments into consideration, the need for greater care for men's sexual health should be emphasized, due to the impact that this disease has on their quality of life. Nonetheless, it must be noted that men's concern about sexual health and their openness to deal with such issues are relatively recent.

\section{REFERENCES}

1. NIH Consensus Conference. Impotence. NIH Consensus Development Panel on Impotence. JAMA-J. Am. Med. Assoc. 1993;270(1):83-90.

2. Monteiro Junior FC. Disfunção erétil: um novo marcador de risco cardiovascular. Revista do Hospital Universitário/UFMA. 2006;7(2):45-6.

3. Walczak MK, Lokhandwala N, Hodge MB, Guay AT. Prevalence of cardiovascular risk factors in erectile dysfunction. J Gend Specif Med. 2002;5(6):19-24.

4. Claudino MA. Influência do treinamento físico na função erétil de ratos com deficiência crônica de óxido nítrico [tese de doutorado]. Campinas: Universidade Estadual de Campinas; 2008.

5. Abdo CHN, Oliveira Jr WM, Scanavino MT, Martins FG. Disfunção erétil - resultados do estudo da vida sexual do brasileiro. Rev Assoc Med Bras. 2006;52(6):424-9.

6. Martínez-Jabaloyas JM. Prevalence of co-morbidities in patients with erectile dysfunction. Actas Urol Es. 2013;37(1):33-9.

7. Sharifi F, Asghari M, Jaberi Y, Salehi O, Mirzamohammadi F. Independent predictors of erectile dysfunction in type 2 diabetes mellitus: is it true what they say about risk factors? Endocrinol. 2012;2012:1-5.

8. Naidan N, Rivaad O, Muukhai N, Janlav M. Testosterone Deficiency with Erectile Dysfunction in Mongolian Men. World J Mens Health. 2013;31(2):170-5. 
9. Javaroni V, Neves MF. Erectile dysfunction and hypertension: impact on cardiovascular risk and treatment. J. Hypertens. 2012;2012:1-11.

10. Wu C, Zhang H, Gao Y, Tan A, Yang X, Lu Z, et al. The association of smoking and erectile dysfunction: results from the fangchenggang area male health and examination survey (FAMHES). J. Androl. 2012;33(1):59-65.

11. Foocharoen C, Tyndall A, Hachulla E, Rosato E, Allanore Y, Farge-Bancel D, Caramasch P, et al. Erectile dysfunction is frequent in systemic sclerosis and associated with severe disease: a study of the eular scleroderma trial and research group. Arthritis Res Ther. 2012;14(1):2-10.

12. Meller SM, Stilp E, Walker CN, Mena-Hurtado C. The link between vasculogenic erectile dysfunction, coronary artery disease, and peripheral artery disease: role of metabolic factors and endovascular therapy. J. Cardiol Invasiva. 2013;25(6):313-19.

13. Banks E, Joshy G, Abhayaratna WP, Kritharides L, Macdonald PS, Korda RJ, et al. Erectile dysfunction severity as a risk marker for cardiovascular disease hospitalisation and all-cause mortality: a prospective cohort study. PLoS Med. 2013;10(1):1-13.

14. Kendirci M, Nowfar S, Hellstrom WJ. The impact of vascular risk factors on erectile function. Drugs Today (Barc). 2005;41(1):65-74.

15. Althof SE, O’ Leary MP, Cappelleri JC, Glina S, King R, Tseng L, et al. Self-esteem, confidence, and relationships in men treated with sildenafil citrate for erectile dysfunction. J Gen Intern Med. 2006;21(10):1069-74.

16. Bayraktar Z, Atun I. Prevalence of self-reported erectile dysfunction among urological cases in turkish men. Urol J. 2011;8(3):214-21.

17. Moreira ED, Abdo CH, Torres EB, Lôbo CF, Fittipaldi JA. Prevalence and correlates of erectile dysfunction: results of the Brazilian study of sexual behavior. Urology. 2001;58(4):583-8.

18. Araujo AB, O'Donnell AB, Brambilla DJ, Simpson WB, Longcope C, Matsumoto $\mathrm{AM}$, et al. Prevalence and incidence of androgen deficiency in middle-aged and older men: estimates from the Massachusetts Male Aging Study. J Clin Endocrinol Metab. 2004;89(12):5920-6.

19. Cairoli CED, Rhoden EL. Disfunção erétil - epidemiologia. In: Wroclawski ER, Bendhack DA, Damião R, Ortiz V. Guia prático de urologia. São Paulo: Segmento, Rio de Janeiro: SBU - Sociedade Brasileira de Urologia, 2003. Seção IIIA,Cap.32.p.115-117.

20. Giami A, Nascimento KC, Russo J. Da impotência à disfunção erétil: destinos da medicalização da sexualidade. Physis. 2009,19(3):637-58.

21. Cheng JYM, Ng EML. Body mass index, physical activity and erectile dysfunction: An U-shaped relationship from population-based study. Int. J. Obes. 2007;31:1571-8.

22. Kałka D, Domagała Z, Dworak J, Womperski K, Rusiecki L, Marciniak W, et al. Association between physical exercise and quality of erection in men with ischaemic heart disease and erectile dysfunction subjected to physical training. Kardiol Pol. 2013;71(6):573-80.

23. La Vignera S, Condorelli R, Vicari E, D'agata R, Calogero A. Aerobic physical activity improves endothelial function in the middle-aged patients with erectile dysfunction. Aging Male. 2011;14(4):265-72.

24. Posch K, Schmidt K, Graier WF. Selective stimulation of L-arginine uptake contributes to shear stress-induced formation of nitric oxide. Life Sci. 1999;64(8):663-70.

25. Esposito K, Giugliano F, Di Palo C,Giugliano G, Marfella R, D’Andrea F, et al. Effect of lifestyle changes on erectile dysfunction in obese men: a randomized controlled trial. JAMA-J. Am. Med. Assoc. 2004;291(24):2978-84.

26. Lim SS, Vos T, Flaxman AD, Danaei G, Shibuya K, Adair-Rohani H, et al.A comparative risk assessment of burden of disease and injury attributable to 67 risk factors and risk factor clusters in 21 regions, 1990-2010: a systematic analysis for the global burden of disease study 2010. Lancet. 2012;380(9859):2224-60.

27. Wang D, He Y, Li Y, Luan D, Zhai F, Yang X, et al. Joint association of dietary pattern and physical activity level with cardiovascular disease risk factors among chinese men: a cross-sectional study. Plos ONE. 2013;8(6):1-28.

28. Donnelly JE, Blair SN, Jakicic JM, Manore MM, Rankin JW, Smith BK. Appropriate physical activity intervention strategies for weight loss and prevention of weight regain for adults. Med Sci Sports Exerc. 2009;41(2):459-71. 
29. Mammen G, Faulkner G. Physical activity and the prevention of depression: a systematic review of prospective studies. Am J Pre Med. 2013;45(5):649-57.

30. Souza CA, Cardoso FL, Silveira RA, Santos MB, Wittkopf PG. Correlação entre capacidade física e atividade sexual em idosos. Rev Bras Geriatr Gerontol. 2011;5(2):80-5.

31. Mirone V, Ricci E, Gentile V, Fasolo CB, Parazzini F. Determinants of Erectile Dysfunction Risk in a Large Series of ItalianMen Attending Andrology Clinics. Eur Urol. 2004;45(1):87-91.

32. Zheng H, Fan W, Li G, Tam T. Predictors for erectile dysfunction among diabetics. Diabetes Res. Clin Pract. 2006;71(3):313-9.

33. Agostini LCM, Netto JMB, Miranda Jr MV, Figueiredo AA. Erectile dysfunction association with physical activity level and physical fitness in men aged 40-75 years. Int J Impot Res. 2011; 23(3):115-21.

34. Urrútia G, Bonfill X. Declaración PRISMA: uma propuesta para mejorar la publicación derevisiones sistemáticas y metaanálisis. Med Clin. 2010;135(11):507-11.

35. Downs SH, Black N. The feasibility of creating a checklist for the assessment of the methodological quality both of randomized and nonrandomized studies of health care interventions. J Epidem Comm Health. 1998;52(52):377-84.

36. Araujo DMR, Vilarim MM, Sabroza AR, Nardi AE. Depressão no período gestacional e baixo peso ao nascer: uma revisão sistemática da literatura. Cad Saúde Pública 2010;26(2): 219-227.

37. Rossi CE, Vasconcelos FAG. Peso ao nascer e obesidade em crianças e adolescentes: uma revisão sistemática. Ver Bras Epidemiol 2010;13(2): 246-258

38. Bacon CG, Mittleman MA, Kawachi I, Giovannucci E, Glasser DB, Rimm EB. A prospective study of risk factors for erectile dysfunction. Journal of urology. 2006;176(1):217-21.

39. Selvin E, Burnett AL, Platz EA. Prevalence and risk factors for erectile dysfunction in the us. Am J Med. 2007;120(2):151-7.

40. Revnic CR, Nica AS, Revnic F. The impact of physical training on endocrine modulation, muscle physiology and sexual functions in elderly men. Arch Gerontol Geriatr. 2007;(Suppl 44):S339-42.

41. Kratzik CW, Lackner JE, Mark I, Rucklinger E, Schmidbauer J, Lunglmayr G, et al. How much physical activity is needed to maintain erectile function? Results of the androx vienna municipality study. Eur Urol. 2009;55:509-17.

42. Araujo AB, Travison TG, Ganz PA, Chiu GR, Kupelian V, Rosen RC, et al. Erectile Dysfunction and Mortality. J Sex Med. 2009;6(9):2445-54.

43. Holden CA, McLachlan RI, Pitts M, Cumming R, Wittert G, Ehsani JP, et al. Determinants of male reproductive health disorders: the Men in Australia Telephone Survey (MATeS). BMC Public Health. 2010;10:1-9.

44. Kupelian V, Araujo AB, Chiu GR, Rosen RC, McKinlay JB. Relative contributions of modifiable risk factors to erectile dysfunction. Results from the Boston Area Community Health (BACH) Survey. Prev Med. 2010;50(1-2):19-25.

45. Pohjantähti-Maaroos H, Palomäki A, Hartikainen J. Erectile dysfunction, physical activity and metabolic syndrome: differences in markers of atherosclerosis. BMC Cardiovasc Disord. 2011;11:1-9.

46. Liao M, Huang X, Gao Y, Tan A, Lu Z, Wu C, et al. Testosterone is associated with erectile dysfunction: a cross-sectional study in Chinese men. PLoS ONE. 2012;7(6):1-8.

47. Garimella PS, Paudel ML, Ensrud KE, Marshall LM, Taylor BC, Fink HA. Association between body size and composition and erectile dysfunction in older men: osteoporotic fractures in men study. J Am Geriatr Soc. 2013;61(1):46-54.

48. Weber MF, Smith DP, O'Connell DL, Patel MI, Souza PL, Sitas F, et al. Risk factors for erectile dysfunction in a cohort of 108477 Australian men. Med J Aust. 2013;199(2):107-11.

49. Nicolosi A, Glasser DB, Moreira ED, Villa M. Prevalence of erectile dysfunction and associated factors among men without concomitant diseases: a population study. Int J Impot Res. 2003a;15(4):253-7.

50. Galvão TFG, Chagas ACP. Disfunção endotelial e fatores de risco. PLAMED. 2005.

51. Coelho OR. Função endotelial, disfunção erétil e doença cardiovascular. PLAMED. 2005. 
52. Eardley I, Sethia K, Dean, J. Disfunção Erétil, parte 1 - Pfiser, dezembro,1998.

53. Nicolosi A, Moreira Jr ED, Shirai M, Tambi MIBM, Glasser DB. Epidemiology of erectile dysfunction in four countries: cross-national study of the prevalence and correlates of erectile dysfunction. Urology. 2003b;61(1):201-6.

54. Leijon ME, Stark-Ekman D, NilsenP, et al. Is there a demand for physical activity interventions provided by the health care sector? Findings from a population survey. 2010. BMC Public Health. 2010; 10:1-8.

\section{CORRESPONDING AUTHOR}

CAMILA DA CRUZ RAMOS DE ARAUJO

Rua Evaldo Schaeffer, 422, bloco B10, Apto

23, Jardim Atlântico, Florianópolis, SC.

Brasil, 88095350. (48)99118140

E-mail: cami.ramosdearaujolahotmail.com 